\title{
Early diagnosis and Early Start Denver Model intervention in autism spectrum disorders delivered in an Italian Public Health System service
}

This article was published in the following Dove Press journal:

Neuropsychiatric Disease and Treatment

14 June 2016

Number of times this article has been viewed

\section{Raffaella Devescovi ${ }^{1}$ \\ Lorenzo Monasta ${ }^{2}$ \\ Alice Mancini ${ }^{3}$ \\ Maura Bin' \\ Valerio Vellante' \\ Marco Carrozzi' \\ Costanza Colombi ${ }^{4}$}

'Division of Child Neurology and Psychiatry, ${ }^{2}$ Clinical Epidemiology and Public Health Research Unit, Institute for Maternal and Child Health - IRCCS "Burlo Garofolo", Trieste, ${ }^{3}$ Department of Clinical and Experimental Medicine, University of Pisa, Pisa, Italy; ${ }^{4}$ Department of Psychiatry, University of Michigan Health System, Ann Arbor, MI, USA
Correspondence: Raffaella Devescovi Division of Child Neurology and Psychiatry, Institute for Maternal and Child Health - IRCCS "Burlo Garofolo", Via dell'Istria 65/I, 34I37 Trieste, Italy

$\mathrm{Tel}+39403785407$

Fax +39 403785544

Email raffaella.devescovi@burlo.trieste.it
Background: Early diagnosis combined with an early intervention program, such as the Early Start Denver Model (ESDM), can positively influence the early natural history of autism spectrum disorders. This study evaluated the effectiveness of an early ESDM-inspired intervention, in a small group of toddlers, delivered at low intensity by the Italian Public Health System.

Methods: Twenty-one toddlers at risk for autism spectrum disorders, aged 20-36 months, received 3 hours/wk of one-to-one ESDM-inspired intervention by trained therapists, combined with parents' and teachers' active engagement in ecological implementation of treatment. The mean duration of treatment was 15 months. Cognitive and communication skills, as well as severity of autism symptoms, were assessed by using standardized measures at pre-intervention (Time 0 [T0]; mean age =27 months) and post-intervention (Time 1 [T1]; mean age =42 months).

Results: Children made statistically significant improvements in the language and cognitive domains, as demonstrated by a series of nonparametric Wilcoxon tests for paired data. Regarding severity of autism symptoms, younger age at diagnosis was positively associated with greater improvement at post-assessment.

Conclusion: Our results are consistent with the literature that underlines the importance of early diagnosis and early intervention, since prompt diagnosis can reduce the severity of autism symptoms and improve cognitive and language skills in younger children. Particularly in toddlers, it seems that an intervention model based on the ESDM principles, involving the active engagement of parents and nursery school teachers, may be effective even when the individual treatment is delivered at low intensity. Furthermore, our study supports the adaptation and the positive impact of the ESDM entirely sustained by the Italian Public Health System.

Keywords: early diagnosis, early intervention, autism spectrum disorder, Early Start Denver Model, Public Health System service

\section{Introduction}

Autism spectrum disorders (ASDs) are a group of neurodevelopmental abnormalities that begin in early childhood and are characterized by impairments in social interaction and communication, as well as repetitive behaviors and restricted interests (Diagnostic and Statistical Manual of Mental Disorders, Fifth Edition [DSM-V]). ${ }^{1}$ Given the high incidence of ASD and the presence of intellectual disabilities and impaired functioning in many individuals with ASD, developing effective interventions that decrease symptoms and improve outcomes is a research and clinical priority. ${ }^{2}$

The role of early intervention in improving children's outcomes is well supported by a consistent body of research. ${ }^{3}$ However, most of the studies supporting early intervention for children with ASDs have been conducted in university centers with 
resources generally greater than those usually available to community services. ${ }^{4}$

One early intervention approach built for toddlers with ASD younger than 3 years is the Early Start Denver Model (ESDM) that combines relationship and developmental theories with teaching techniques based on the principle of behavioral analysis. ${ }^{5}$ In the ESDM, rigorous teaching, based on individualized objectives, occurs within the framework of playful activities.

While the positive effects of the ESDM are well documented by previous studies, ${ }^{4-6}$ the feasibility and the efficacy of the model in day community care services need to be evaluated.

Evaluating evidence-based interventions in community settings is currently a step needed in the intervention research realm to ensure that the models developed in university settings are feasible and sustainable in services not supported by research funds.

Here, we propose a retrospective study of toddlers at risk for ASD, presenting our experience on the feasibility and effectiveness of the ESDM intervention delivered within the childhood and adolescent psychiatric services offered by the Italian Public Health System.

\section{Methods}

We retrospectively evaluated a group of toddlers at risk for ASD recruited at the Division of Child Neurology and Psychiatry of the Institute for Maternal and Child Health IRCCS "Burlo Garofolo" in Trieste, Italy, a regional public institute for health care and scientific research. All parents had given written consent to the use of data collection and analysis for scientific purposes, and the study was approved by the Technical Scientific Committee of the Institute for Maternal and Child Health - IRCCS "Burlo Garofolo", Trieste.

Assessment and intervention were provided by distinct staff since treatment is usually delivered in community centers specialized for childhood and adolescence, as established by the Italian Public Health System.

The entire sample was therefore evaluated by blinded clinicians operating in the Division of Child Neurology and Psychiatry of IRCCS "Burlo Garofolo" at the beginning (T0) and at the end of intervention (T1). The assessment was conducted with standardized measures of cognitive and language skills and ASD symptoms. The time elapsed between $\mathrm{T} 0$ and $\mathrm{T} 1 \mathrm{ranged}$ from 11 months to 19 months, with a median of 15 months and an interquartile range (IQR) of 12-16 months.

\section{Participants}

The sample consisted of 21 children, 18 males and three females, with age ranging from 20 months to 36 months at T0 (median 27 months; IQR 22-30 months) and from 31 months to 61 months at T1 (median 42 months; IQR 39-48 months). All children received ASD diagnosis based on clinical observation and according to $D S M-V .{ }^{1}$ Diagnosis was confirmed by the Autism Diagnostic Observation Schedule-Second Edition (ADOS-2) ${ }^{7}$ administered by experienced clinicians trained for research reliability. It was the children's first diagnosis, and none had received any type of treatment previously.

The exclusion criteria applied were known neurodevelopmental disorders of known genetic etiology (eg, Fragile $\mathrm{X}$ syndrome), neurological disorders (eg, epilepsy), and significant vision, hearing, motor, or physical problems.

\section{Measures}

\section{Developmental functioning}

For cognitive and language skills, the Bayley Scales of Infant and Toddler Development, Third Edition (Bayley-III), ${ }^{8}$ a developmental assessment for children between 0 months and 42 months, was administered. When children increased in age over 42 months, the Wechsler Preschool and Primary Scale of Intelligence, Third Edition (WPPSI-III) ${ }^{9}$ was used. The WPPSI-III scale provides verbal intelligence quotient (IQ) and performance IQ scores, as well as full-scale IQ scores. In order to compare the different scales, we established correlations between the WPPSI-III IQ scores and the composite scores of Bayley-III Cognitive and Language scales, which were 0.81 and 0.78 , respectively, according to Bode et al. ${ }^{10}$ Therefore, when the WPPSI-III scale was administered at T1, we considered the composite scores of Bayley-III Cognitive and Language scales equal to the fullscale IQ and verbal IQ scores of the WPPSI-III scale.

\section{Autism severity}

The ADOS-2 is a semistructured interaction schedule that measures symptoms of autism in social communication, play, and repetitive behaviors. ${ }^{7}$ It provides an empirically derived algorithm that differentiates children with ASD from those with other delays or typical development, thus supporting the clinical judgment in diagnosis. To evaluate changes in autism severity from $\mathrm{T} 0$ to $\mathrm{T} 1$, in accordance with Gotham et al, ${ }^{11}$ as a variable in statistical analysis, we used the calibrated severity score, a standardized system of calculating ASD symptom severity from ADOS-2 scores ranging from 1 to 10 , with higher scores indicating greater ASD symptom severity.

For the nine children who were assessed by the ADOSToddler Module, ${ }^{12}$ Module 1 algorithms and severity scores were calculated according to the well-based procedures described by Guthrie et al, ${ }^{13}$ so that the severity of autistic symptoms could be defined for all children. 


\section{Intervention}

The ESDM focuses on the core areas of ASD impairment: verbal and nonverbal communication, joint attention, social engagement, imitation, play, and cognition..$^{5}$ The ESDM is delivered within the context of play and daily routines in which highly precise teaching is embedded. For each child, an individualized intervention was developed to include a variety of objectives from all areas of development, including communication, motor development, cognition, social interaction, and daily living skills, in accordance with the ESDM curriculum and teaching principles.

Each child received 3 hours/wk of one-to-one sessions of the ESDM-inspired treatment, delivered in a dedicated community center, for $\sim 15$ months. The treatment team included professionals with background in behavioral principles, clinical and developmental psychology, speech and language pathology, and occupational therapy. Prior to the beginning of the intervention, all therapists participated in the Introductory and Advanced ESDM Training modules. The team was supervised by a certified ESDM trainer and worked toward fidelity of implementation during the intervention. Fidelity was reached after the end of the intervention. Therefore, we refer to the intervention as ESDM inspired rather than ESDM. The clinicians discussed treatment objectives and
ESDM strategies with parents during the sessions, as well as with day care and preschool teachers on a monthly basis.

\section{Statistical analysis}

Data were summarized as frequencies and percentages or as medians and IQRs depending on the nature of the variables. Wilcoxon signed-rank tests for paired data were applied to verify if there were significant differences between scores at $\mathrm{T} 0$ and $\mathrm{T} 1$.

Logistic regression was used to study the association between improvement in developmental functioning and autism symptoms severity and chronological age at diagnosis. For all analyses, a $P$-value $<0.05$ was considered as statistically significant. All statistical analyses were carried out with Stata/IC 14.1 (StataCorp LP, College Station, TX, USA).

\section{Results}

According to the signed-rank test, a significant improvement in the entire sample was found in both the cognitive and language domains comparing $\mathrm{T} 0$ and $\mathrm{T} 1$ results (respectively, $P=0.0269$ and $P=0.0254$ ). The cognitive domain scores rose from a median of 77.5 (IQR 65-90) to 90 (IQR 73-95), while the language domain score increased from 56 (IQR 50-68) to 74 (IQR 56-94; Figure 1A and B).
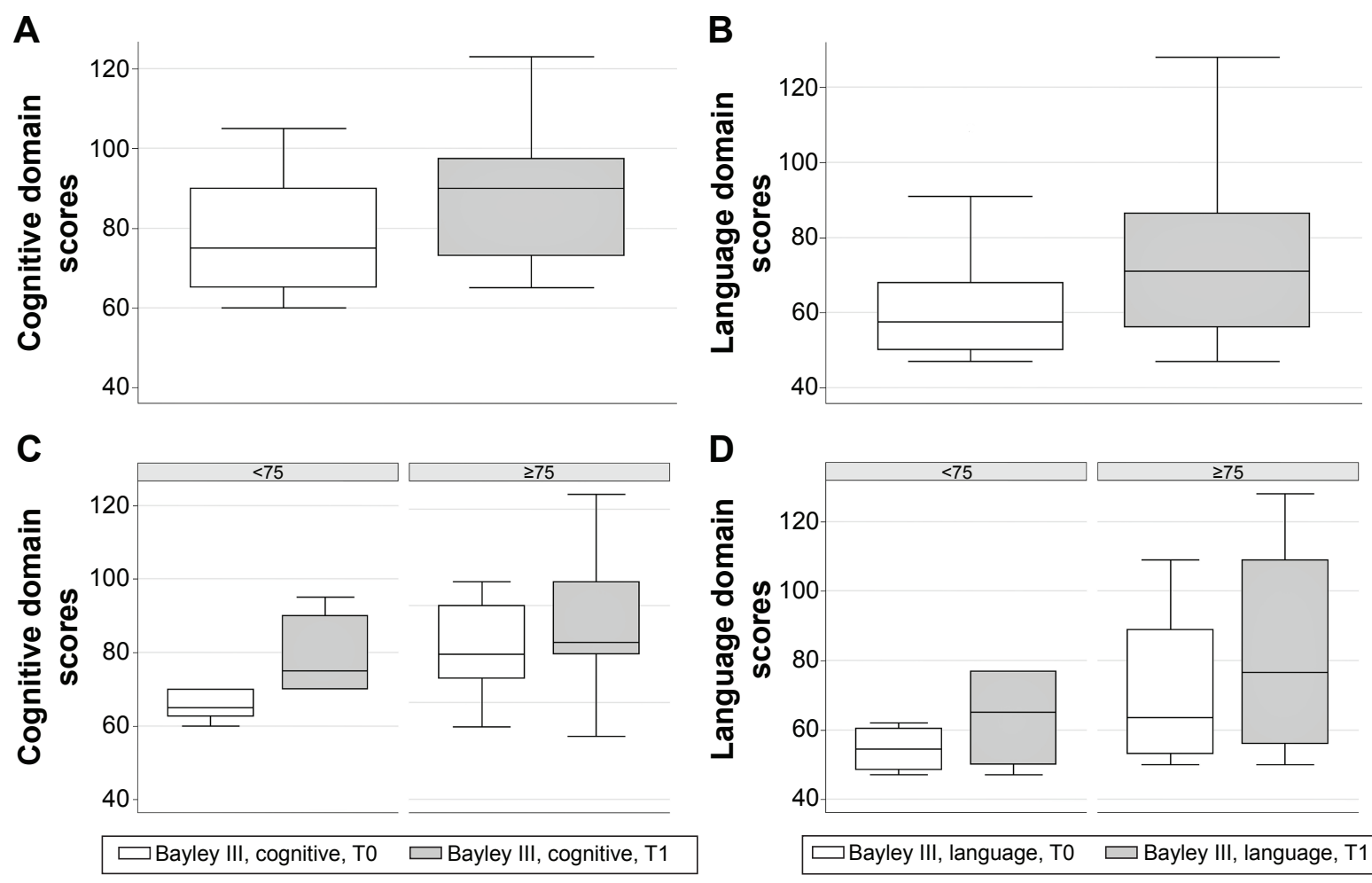

Figure I Box plots of cognitive domain scores and language domain scores at baseline (T0) and after treatment (TI).

Notes: (A and B) Entire group. (C and D) The sample stratifies into two groups based on the cognitive scale score at T0 ( $<75$ and $\geq 75)$. The whiskers extend two-thirds the width of a box unless the minimum or the maximum values are closer to the median.

Abbreviation: Bayley-III, Bayley Scales of Infant and Toddler Development, Third Edition. 
Then, we stratified the sample into two groups based on the cognitive scale score at $\mathrm{T} 0$, separating those with a score $<75$ from those with $\geq 75$. Notably, the first group $(<75)$ showed a significant increase in the cognitive and language scale scores $(P=0.0218$ and $P=0.0496)$, while for the second group ( $\geq 75$ ), the increase was not significant $(P=0.3669$ and $P=0.1610$; Figure $1 \mathrm{C}$ and $\mathrm{D})$.

Regarding the ADOS severity scores, we found no significant difference in the entire group between pre- and post-interventions $(P=0.3636)$, although a reduction in the severity of symptoms can be appreciated from Figure 2A. Also, no significant difference had been found, after the stratification, in the group with cognitive scale score $<75$ ( $P=0.881$; data not shown).

However, observing the data distribution, we realized that most children younger than 27 months at T0 made the biggest progress in reducing severity of ASD symptoms after intervention. Thus, a logistic regression analysis was performed considering the improvement in the ADOS severity score as a dependent variable. Age was also dichotomized $(0,<27$ months; $1, \geq 27$ months) and entered as an independent variable in the model. We obtained a significant association between early diagnosis and improvement in autistic symptoms (odds ratio $=15.0 ; P=0.028$ ), suggesting that a child who was younger than 27 months at T0 had 15 times more chance of improving his/her ADOS severity score than a child who was 27 months or older at T0 (Figure 2B).

\section{Discussion}

Our findings suggest that a group of preschool-aged children with ASD, treated with a low-intensity ESDM-inspired intervention, delivered by an Italian Public Health System, presented statistically significant postintervention improvements in language and cognitive functioning and a decrease in autism severity, although without significant improvements in the ADOS severity scores.

Our study supports early screening and intervention for ASD to promote better outcomes, which is in accordance with the study of Howlin et a ${ }^{14}$ who found that chronological age at baseline was significantly associated with improvement in the ADOS severity scores.

Moreover, in our study, the regression analysis suggests that children who receive diagnosis earlier than 27 months of age have better prognosis as to severity of autism symptoms.

The use of an evidence-based intervention to promote best outcomes, specifically developed for young children with ASD, such as the ESDM, is well supported in the research literature. For example, Dawson et $\mathrm{al}^{6}$ and Vivanti et $\mathrm{al}^{4}$ suggest that interventions specifically developed for very young children with ASD lead to better outcomes in comparison to generic community-based interventions. In these studies, the children received 20 hours/wk of one-to-one ESDM intervention by trained therapists in a university clinic setting, while our study was conducted within the Italian Public Health System, in dedicated community centers, where it can be offered to the children only as low-intensity treatment, because of obvious resource limitations.

Despite less hours of direct therapist involvement, we presume that the active engagement of parents and teachers in the treatment process, implementing the strategies in natural daily routines, has positively influenced the outcome. There is no certainty on long-term outcome from the literature,
A

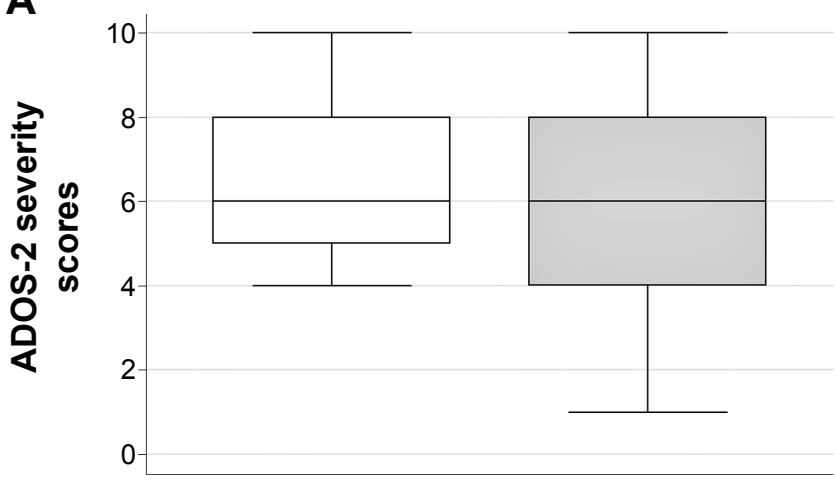

B

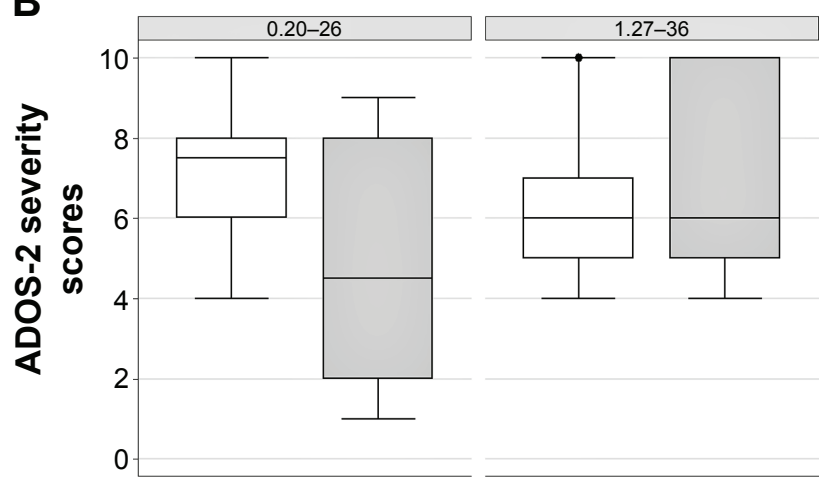

ADOS, T0 $\square$ ADOS, T1

Figure 2 Box plots of the ADOS-2 scores at baseline (T0) and after treatment (TI).

Notes: (A) Entire group. (B) The sample stratifies into two groups based on age at T0 ( $<27$ months and $\geq 27$ months).

Abbreviation: ADOS-2, Autism Diagnostic Observation Schedule - Second Edition. 
even though recent studies support the clinical data that positive effects can be obtained with fewer hours of individual intervention by emphasizing the central role of parents or by delivering ESDM intervention in a community child care setting. ${ }^{15,16} \mathrm{We}$ are aware that our study has the limitation of being a retrospective analysis that did not include a control group without intervention or other early treatment. Of course, lacking a control group, we must assume that the gains observed from $\mathrm{T} 0$ to $\mathrm{T} 1$ are part of the natural evolution of ASD and owed to maturation rather than to the intervention itself. However, our knowledge about the natural history of autistic disorders is supported by a previous study that describe a diagnostic stability based on clinical judgment, with $80 \%$ of children remaining in the same diagnostic category at follow-up and only $20 \%$ with changes in diagnosis to a nonautistic developmental disorder or to no diagnosis. ${ }^{17}$

Furthermore, we observed a significant gain in IQ that cannot be attributed to only maturational effects. In fact, a vast majority of studies state that IQ remains invariable over time, such as in a review of 23 longitudinal studies in children with ASD. ${ }^{18,19}$

According to these studies, spontaneous improvement is observed mostly in children with initial higher functioning and less severe autism, whereas children with more severe clinical features at onset often show a developmental regression.

Remarkably, in our study, the children who seemingly benefited most from the early treatment were those with a lower IQ at the first assessment. In our opinion, it is unlikely that cognitive and language improvement, as well as less severe autistic symptoms, observed in our sample can be attributed to maturational effects alone and not consequent to the treatment or to the combined effects of both. We thus agree with Vivanti et $\mathrm{al}^{20}$ that low cognitive level at the first assessment is not a comorbid feature of autism but a consequence of the social-communication deficits on neurocognitive development. This interpretation emphasizes the importance of early exposure to adequate social stimulation in order to support the child's cognitive development.

From an ethical point of view, our results of significant clinical improvements first emphasize the need for early detection of children "at risk" of autism and second call for an early child care program, feasible for implementation by the Public Health System and available to all the children with ASD.

\section{Conclusion}

Although these results need to be confirmed by a control group study and a larger sample, we believe that ESDM intervention, provided during a very critical and sensitive period of development, may be feasible and effective even when delivered at low intensity.

\section{Acknowledgments}

The authors thank Laura Bravar for careful language revision of the manuscript. We are grateful to the group of the therapists who delivered the ESDM treatment in public services. The group was composed of psychologists and rehabilitation therapists trained in the ESDM method who are listed in alphabetical order: Antonella Celea, Boris Cerniz, Cesarino Zago, Chiara Terpini, Daniela Vidoni, Devid Cescon, Francesca Placer, Linda Puzzer, Marcella Guerrieri, Monica Stocchi, and Sara Frisari.

\section{Disclosure}

The authors report no conflicts of interest in this work.

\section{References}

1. American Psychiatric Association. Diagnostic and Statistical Manual of Mental Disorders. 5th ed. Arlington, VA: American Psychiatric Publishing; 2013.

2. Baxter AJ, Brugha TS, Erskine HE, Scheurer RW, Vos T, Scott JG. The epidemiology and global burden of autism spectrum disorders. Psychol Med. 2015;45(3):601-613.

3. Dawson G, Jones EJ, Merkle K, et al. Early behavioral intervention is associated with normalized brain activity in young children with autism. J Am Acad Child Adolesc Psychiatry. 2012;51(11): $1150-1159$.

4. Vivanti G, Paynter J, Duncan E, et al. Effectiveness and feasibility of the early start denver model implemented in a group-based community childcare setting. J Autism Dev Disord. 2014;44(12):3140-3153.

5. Rogers SJ, Dawson G. The Early Start Denver Model for Young Children with Autism: Promoting Language, Learning, and Engagement. New York: Guilford Press; 2010.

6. Dawson G, Rogers S, Munson J, et al. Randomized, controlled trial of an intervention for toddlers with autism: the Early Start Denver Model. Pediatrics. 2010;125(1):e17-e23.

7. Lord C, Rutter M, DiLavore PC, Risi S, Gotham K, Bishop S. Autism Diagnostic Observation Schedule, Second Edition (ADOS-2) Manual (Part I): Modules 1-4. Torrance, CA: Western Psychological Services; 2012.

8. Bayley N. Bayley Scales of Infant and Toddler Development: Bayley-III San Antonio, TX: Harcourt Assessment; 2006.

9. Wechsler D. Wechsler Preschool and Primary Scale of Intelligence Third Edition WPPSI III. Florence: Giunti O.S; 2008.

10. Bode MM, D'Eugenio DB, Mettelman BB, Gross SJ. Predictive validity of the Bayley, Third Edition at 2 years for intelligence quotient at 4 years in preterm infants. $J$ Dev Behav Pediatr. 2014;35(9):570-575.

11. Gotham K, Risi S, Pickles A, Lord C. The Autism Diagnostic Observation Schedule: revised algorithms for improved diagnostic validity. J Autism Dev Disord. 2007;37(4):613-627.

12. Lord CE, Luyster R, Gotham K, Guthrie WJ. Autism Diagnostic Observation Schedule - Toddler Module. Los Angeles, CA: Western Psychological Services; 2012

13. Guthrie W, Swineford LB, Nottke C, Wetherby AM. Early diagnosis of autism spectrum disorder: stability and change in clinical diagnosis and symptom presentation. $J$ Child Psychol Psychiatry. 2013;54(5): $582-590$. 
14. Howlin P, Moss P, Savage S, Rutter M. Social outcomes in mid- to later adulthood among individuals diagnosed with autism and average nonverbal IQ as children. J Am Acad Child Adolesc Psychiatry. 2013;52(6): e1.572-e1.581.

15. Green J, Charman T, McConachie H, et al. PACT Consortium. Parent-mediated communication-focused treatment in children with autism (PACT): a randomised controlled trial. Lancet. 2010;40(9):1154-1160.

16. Eapen V, Crnčec R, Walter A. Clinical outcomes of an early intervention program for preschool children with Autism Spectrum Disorder in a community group setting. BMC Pediatr. 2013;13(1):3.

17. Kleinman JM, Ventola PE, Pandey J, et al. Diagnostic stability in very young children with autism spectrum disorders. J Autism Dev Disord. 2008;38(4):606-615.
18. Begovac I, Begovac B, Majić G, Vidovic V. Longitudinal studies of IQ stability in children with childhood autism - literature survey. Psychiatr Danub. 2009;21(3):310-319.

19. Eaves LC, Ho HH. The very early identification of autism: outcome to age 4 1/2-5. J Autism Dev Disord. 2004;34(4):367-378.

20. Vivanti G, Barbaro J, Hudry K, Dissanayake C, Prior M. Intellectual development in autism spectrum disorders: new insights from longitudinal studies. Front Hum Neurosci. 2013;7:354.

\section{Publish your work in this journal}

Neuropsychiatric Disease and Treatment is an international, peerreviewed journal of clinical therapeutics and pharmacology focusing on concise rapid reporting of clinical or pre-clinical studies on a range of neuropsychiatric and neurological disorders. This journal is indexed on PubMed Central, the 'PsycINFO' database and CAS, and is the official journal of The International Neuropsychiatric Association (INA). The manuscript management system is completely online and includes a very quick and fair peer-review system, which is all easy to use. Visit http://www.dovepress.com/testimonials.php to read real quotes from published authors.

Submit your manuscript here: http://www.dovepress.com/neuropsychiatric-disease-and-treatment-journal 